\title{
Super-Eddington Accretion in the Ultraluminous X-ray Source NGC1313 X-2: An Ephemeral Feast
}

\author{
Shan-Shan Weng ${ }^{1,2,3}$, Shuang-Nan Zhang ${ }^{2,4,5}$, Hai-Hui Zhao ${ }^{2}$ \\ wengss@ihep.ac.cn; zhangsn@ihep.ac.cn; zhaohh@ihep.ac.cn
}

\begin{abstract}
We investigate the X-ray spectrum, variability and the surrounding ionized bubble of NGC1313 X-2 to explore the physics of super-Eddington accretion. Beyond the Eddington luminosity, the accretion disk of NGC1313 X-2 is truncated at a large radius ( $\sim 50$ times of innermost stable circular orbit), and displays the similar evolution track with both luminous Galactic black-hole and neutron star X-ray binaries. In super-critical accretion, the speed of radiatively driven outflows from the inner disk is mildly relativistic. Such ultra-fast outflows would be over ionized and might produce weak Fe K absorption lines, which may be detected by the coming X-ray mission Astro-H. If the NGC1313 X-2 is a massive stellar X-ray binary, the high luminosity indicates that an ephemeral feast is held in the source. That is, the source must be accreting at a hyper-Eddington mass rate to give the super-Eddington emission over $\sim 10^{4}-10^{5} \mathrm{yr}$. The expansion of the surrounding bubble nebula with a velocity of $\sim 100 \mathrm{~km} \mathrm{~s}^{-1}$ might indicate that it has existed over $\sim 10^{6} \mathrm{yr}$ and is inflated by the radiatively driven outflows from the transient with a duty cycle of activity of $\sim$ a few percent. Alternatively, if the surrounding bubble nebula is produced by line-driven winds, less energy is required than the radiatively driven outflow scenario, and the radius of the Strömgren radius agrees with the nebula size. Our results are in favor of the line-driven winds scenario, which can avoid the conflict between the short accretion age and the apparently much longer bubble age inferred from the expansion velocity in the nebula.
\end{abstract}

\footnotetext{
${ }^{1}$ Department of Physics, Xiangtan University, Xiangtan 411105, China

${ }^{2}$ Key Laboratory of Particle Astrophysics, Institute of High Energy Physics, Chinese Academy of Sciences, Beijing 100049, China

${ }^{3}$ Sabancı University, Faculty of Engineering and Natural Sciences, Orhanlı- Tuzla, İstanbul 34956, Turkey

${ }^{4}$ National Astronomical Observatories, Chinese Academy of Scienc es, Beijing, 100012, China

${ }^{5}$ Physics Department, University of Alabama in Huntsville, Huntsville, AL 35899, USA
} 
Subject headings: accretion, accretion disks — black hole physics — X-rays: binaries - X-rays: stars - X-rays: individual (NGC1313 X-2)

\section{Introduction}

Hundreds of non-nuclear X-ray sources have been found in nearby galaxies with isotropic luminosities $>10^{39} \mathrm{erg} / \mathrm{s}$, now called ultraluminous X-ray sources (ULXs, Liu \& Mirabel 2005; Swartz et al. 2011). Recently, Feng \& Soria (2011) reviewed the multiwavelength studies of ULXs, and summarized that ULXs are a diverse population (see also Fender \& Belloni 2012). A handful of ULXs contain normal stellar mass black holes (BHs), with a mass $M_{\mathrm{BH}} \sim 5-15 M_{\odot}$ (Weng et al. 2009; Middleton et al. 2012). A few brightest ULXs are strong candidates of intermediate mass BHs (IMBHs), $M_{\mathrm{BH}} \sim 10^{2}-10^{4} M_{\odot}$, e.g. ESO24349 HLX-1 (Farrell et al. 2009; Webb et al. 2012) and M82 X-1 (Strohmayer \& Mushotzky 2003). The majority of ULXs are thought to be massive stellar black hole X-ray binaries $(\mathrm{MXBs}), M_{\mathrm{BH}} \sim 50-100 M_{\odot}$ (Patruno \& Zampieri 2008).

The mass of a BH depends both on the initial mass and metallicity of stellar progenitor (Heger et al. 2003; Zampieri \& Roberts 2009; Belczynski et al. 2010). The low metallicity environments in the early universe are suitable for massive stars producing massive BHs. The role of MXBs in cosmology has attracted increasing attention in recent years (e.g. Wheeler \& Johnson 2011; Fragos et al 2013; Power et al. 2013). Mirabel et al. (2011) argued that besides the ultraviolet (UV) radiation from massive stars, feedback from MXBs is an additional, important source of heating and reionization of the Inter Galactic Medium at the dawn of the universe. Justham \& Schawinski (2012) suggested that the MXB feedback might significantly affect the galaxy formation, especially the dwarf galaxies and the earliest epoch of galaxy formation. If most ULXs are MXBs, they are a good sample for us to study all these physical processes. The luminosities $\sim 10^{40} \mathrm{erg} / \mathrm{s}$ illustrates super-Eddington accretion in this population of ULXs (Gladstone et al. 2009; Weng et al. 2009).

However, due to various reasons, our understanding of super-Eddington accretion flow is extremely rudimentary. There is a subclass of active galactic nuclei (AGNs) that are candidates for super-Eddington accretion, namely, narrow line Seyfert 1 galaxies (Collin \& Kawaguchi 2004; Middleton \& Done 2010). However, such sources are far away from the earth, and their environments are relatively complicated. Collecting a few bright Galactic sources, Weng \& Zhang (2011, Paper I) found that BH and neutron star (NS) X-ray binaries (XRBs) shared the same accretion disk evolution pattern when approaching Eddington luminosity (see also Neilsen et al. 2011). Through the observed NS surface emission, in Paper I we argued that the disk thickness $\mathrm{H} / \mathrm{R}$ is less than $0.3-0.4$, and the significant outflow from 
inner disk edge exists at luminosity close to Eddington luminosity.

NGC1313 X-2 is one of the best-studied ULXs, which has the richest observation data at both X-ray and optical wavelengths (Liu et al. 2007; Pintore \& Zampieri 2012). The source was first detected by Einstein observatory (Fabbiano \& Trinchieri 1987), and then observed with many other X-ray missions. Its apparent X-ray luminosity varies between a few $10^{39} \mathrm{erg} / \mathrm{s}$ and $3 \times 10^{40} \mathrm{erg} / \mathrm{s}$ in $0.3-10.0 \mathrm{keV}$ (Feng \& Kaaret 2006; Mizuno et al. 2007), making it a typical ULX. The X-ray spectrum of NGC 1313 X-2 shows a conventionally smooth and featureless profile, which can be modeled in different ways (Stobbart et al. 2006). Many observations suggest that NGC1313 X-2 is a MXB system, with a BH mass $M_{\mathrm{BH}} \sim 50-100 M_{\odot}$ and a companion star $M_{\mathrm{MS}} \sim 8-20 M_{\odot}$ (Zampieri et al. 2004; Grisé et al. 2008; Liu et al. 2012). Zampieri et al. (2004) investigated the X-ray properties with Chandra, ROSAT, ASCA, and XMM-Newton data, and suggested that the mass of the compact remnant is $\sim 100 M_{\odot}$. The optical luminosity derived from ESO data indicated that the mass of the companion is $\sim 15-20 M_{\odot}$ (Zampieri et al. 2004). Grisé et al. (2008) analysed the VLT/FORS1 and HST/ACS photometric data, found that NGC1313 X-2 is associated with a star cluster with an age of $20 \mathrm{Myr}$, and placed an upper mass limit of some $12 M_{\odot}$ for the companion. Using the constraints from the optical observations, Patruno \& Zampieri $(2008,2010)$ computed evolution tracks of ULX counterparts on the color-magnitude diagram, and restricted NGC1313 X-2 to be a 50-100 $M_{\odot}$ BH accreting from a $12-15 M_{\odot}$ main-sequence star. Liu et al. (2009) firstly reported a possible orbital period $P \sim 6.12$ days from the source with HST observations. They also constrained the binary masses and other parameters for NGC1313 X-2 by fitting the detected light curves and radial velocity changes of the disk emission lines to an X-ray irradiated binary model (Liu et al. 2012). However, the significance of this period was low (Zampieri et al. 2012), and further spectroscopic monitoring observations are required to firmly determine both the period and the mass function of NGC1313 X-2. Of special interest is the surrounding ionized bubble nebula ( 200 pc, Pakull \& Mirioni 2002), resembling ordinary supernova remnants, but an order of magnitude larger. It is suggested that the continuous outflow from the central X-ray source blows up the bubble (Pakull et al. 2006).

In this work, we attempt to use XMM-Newton archive data and the information of ionized bubble nebula taken with ESO VLT, HST, and the SUBARU telescope, to address the super-critical accretion flow in the following questions. (1) How does the accretion disk evolve when the source is in super-Eddington luminosity? (2) Can the source give the superEddington emission for a long time? (3) Does the bubble nebula result from the outflow formed in highly super-critical accretion? We describe the spectral and timing analyses in next two sections, make the discussion in Section 4, and finally present the conclusion in Section 5 . 


\section{Spectral Analysis}

We analyze all of the available XMM-Newton observations of NGC1313 X-2, which were made prior to 2007. The data files from the EPIC camera are reduced with the standard tools of XMM-SAS software version 11.0.0.. The light curve above $10 \mathrm{keV}$ is created, and then a count rate cut-off criterion is used to exclude background flares. We select the data from good time intervals, by setting FLAG $=0$ and PATTERN $\leq 4$ for PN data, and PATTERN $\leq 12$ for MOS data. Source spectra are extracted from circles with radius of $32^{\prime \prime}$ and centered at the nominal position of NGC1313 X-2 (RA = 03:18:22.3, Dec $=-66: 36: 03.8$, J2000), while background spectra from the same CCD chips as the source and at a similar distance from the readout node. The spectral response file are created using the SAS task rmfgen and arfgen. In several cases, PN or MOS data are unavailable because the source was not covered by the detector, or due to CCD gaps. When available, PN and MOS data from each individual observation are fitted together for spectral analysis. The $0.3-10.0 \mathrm{keV}$ band spectra are fitted with the HEAsoft X-ray spectral fitting package XSPEC 12.7.1. All spectra are rebinned to have at least 20 counts per bin to enable the use of $\chi^{2}$ statistics.

We first fit the spectra with a powerlaw model, and a multicolor disk blackbody model (diskbb in XSPEC) is added if it has a significance level above $99 \%$ based on F-Test (Feng \& Kaaret 2006). All models in the paper also include the interstellar absorption with the absorption column density being treated as a free parameter. The unabsorbed total flux and the disk flux in $0.3-10.0 \mathrm{keV}$ are calculated with the convolution model cflux. The fitting results are shown in Table 1.

Unlike the cases in canonical low/hard and high/soft states in XRBs, ULXs present no clear gaps, but a broad continuous distribution in photon index $(\Gamma \sim 1$.0-3.0, Feng \& Soria 2011). In NGC1313 X-2, the photon index does not correlate with the disk flux, nor the total flux (Tables 1-3). In 8 of 14 observations, the source exhibits a significant disk component, and its inner disk radius can be estimated from the diskbb model as:

$$
R_{\text {disk }}=\xi_{\text {cor }} N_{\text {disk }}^{1 / 2} \frac{D}{10 \mathrm{kpc}} \cos \theta^{-1 / 2} f_{\text {col }}^{2} \mathrm{~km},
$$

where $N_{\text {disk }}$ is the normalization, the distance $D=4.13 \mathrm{Mpc}$ (Méndez et al. 2002), $\theta$ is the inclination angle of the disk, $f_{\text {col }}$ is the fractional change of the color temperature and $\xi_{\text {cor }}$ is the correction factor for the inner torque-free boundary condition (Zhang et al. 1997). With the fitted inner disk radius $R_{\text {in }}$ and its temperature $T_{\text {in }}$, the bolometric luminosity of the disk can also be derived as: $L_{\text {disk }}=4 \pi R_{\mathrm{in}}^{2} \sigma_{\mathrm{SB}} T_{\mathrm{in}}^{4}$. The Eddington luminosity is $L_{\text {Edd }}=1.3 \times 10^{38} \times M / M_{\odot}$ erg s${ }^{-1}$ with $M$ being the mass of central compact object. Assuming the $\mathrm{BH}$ mass is $50 M_{\odot}$, and a set of reasonable parameters (i.e., $\theta=70^{\circ}, \xi_{\text {cor }}$ $=0.41$ (Kubota et al. 1998), and $f_{\text {col }}=1.7$ ), we plot the ISCO-scaled radius of the inner 
accretion disk versus the bolometric luminosity of the disk in units of $L_{\text {Edd }}$ in Figure 1(a), where the Galactic XRBs 1 data are also shown (see Paper I and references therein).

It is interesting that the behavior of NGC1313 X-2 just resembles those of the Galactic $\mathrm{XRBs}$ in the high-luminosity tail. However, in such cool disk model, the disk contribution is overwhelmed by a powerlaw component, which may possibly come from the Comptonization of blackbody seed photons (Yao et al. 2005). Using the simple powerlaw to depict the Compton component also introduces another problem: it rises without limit at low energies, which evidently disagrees with Comptonization. In this way, the flux of powerlaw component is overestimated, and the flux of the soft thermal (diskbb) component is suppressed, i.e. its normalization is reduced. That is, the fits with diskbb+powerlaw give a cooler and smaller disk component than reality (Yao et al. 2005; Steiner et al. 2009). Therefore, before deriving the nature of the source, a physical Comptonization model is needed.

Here we use an empirical model of Comptonization, SIMPL ( simpl in XSPEC), to fit all 14 observations (Table 1 and Figure 1(b)). SIMPL offers a generic approach to fitting Comptonized spectra with only two free parameters - a photon index and a scattered fraction of input seed photons. This model is valid for a broad range of geometric configurations, and successfully matches the behavior of other physical Comptonization models (see text and references in Steiner et al. 2009). However, the fits fail to converge in 4 of 14 observations, and the errors of some parameters in those fits are unavailable (Table 1). Compared with those cases in powerlaw model, the diskbb component in simpl model contributes a larger fraction of X-ray emission in $0.3-10.0 \mathrm{keV}$, even exceeds the non-thermal component (Tables 1-3). The bolometric luminosity of disk component is up to several times of the non-thermal emission in $0.3-10.0 \mathrm{keV}$ in simpl model. However, we cannot obtain reliable information of non-thermal emission, and can hardly extrapolate its bolometric luminosity with the XMMNewton's limited observing window (0.3-10.0 keV). Bachetti et al. (2013) reported that NGC1313 X-2 is not significantly detected by $N u S T A R$ above $10 \mathrm{keV}$, suggesting that the bolometric luminosity of NGC1313 X-2 is dominated by the disk component.

Because the disk component peaks where the interstellar absorption is of high importance, the fitted disk flux is influenced by the absorption column density $N_{\mathrm{H}}$ (Feng \& Kaaret 2007). Unfortunately, it is unclear whether the variation of absorption column density in difference orbital phase is true. In this way, we carry out a series of tests before obtaining the final results.

1. We replace the wabs model with another absorption model, e.g., the TuebingenBoulder interstellar medium (ISM) absorption model (tbabs in XSPEC), and the results of

\footnotetext{
${ }^{1}$ In this paper, we use the term "Galactic XRBs" to include also LMC X-3.
} 
tbabs* (diskbb+po) are shown in Table 2. However we fail to constrain some parameters with tbabs* ${ }^{*} i m p l * d i s k b b$ model, similar to the case with wabs $^{*} \operatorname{simpl}{ }^{*}$ diskbb. The value of $N_{\mathrm{H}}$ is insensitive to absorption models used, and the fitting results of tbabs model are very similar to (or with slightly higher $N_{\mathrm{H}}$ than) the results obtained with wabs model.

2. Feng \& Kaaret (2007) created two-dimensional plots of the $1 \sigma$ confidence contours (model of $w a b s^{*}(d i s k b b+p o)$ ), allowing both $N_{\mathrm{H}}$ and $k T_{\text {disk }}$ to vary (figure 1 in their paper). Taking the influence of $N_{\mathrm{H}}$ into account, the anti-correlation between $L_{\text {disk }}$ and $T_{\text {disk }}$ did not change but with larger scatter.

3. Assuming that $N_{\mathrm{H}}$ does not change among 14 observations, we fit all observations simultaneously with the same value of $N_{\mathrm{H}}$, but leave the other parameters untied (Table 3 and Figure 1(c)). In this way, the disk flux of some observations are modified significantly; nevertheless, the data remain in the sequence presented in the inner disk radius-luminosity plane, and our discussion and conclusion are still valid.

Note that to obtain the accurate values of $R_{\text {disk }}$ and $L_{\text {disk }}$, we need not only high quality X-ray data, but also the knowledge of $\xi_{\text {cor }}, f_{\text {col }}, D$, and $\theta . R_{\text {disk }}$ and $L_{\text {disk }}$ are related to these parameters: $R_{\text {disk }} \propto \xi_{\text {cor }} f_{\text {col }}^{2} D \cos \theta^{-0.5}$ and $L_{\text {disk }} \propto D^{2} \cos \theta$. On the other hand, $R_{\mathrm{ISCO}}$ is inversely correlated with the spin $a_{*}$ (Bardeen et al. 1972; Zhang et al. 1997). Unfortunately, we cannot constrain these parameters well from any existing observation. With the same parameters as those of XTE J1701-462, the NGC1313 X-2 disk evolution sequence is parallel to and above the sequence of high-luminosity Galactic XRBs. Changing the mass of $\mathrm{BH}$ decreases both $R_{\text {disk }} / R_{\mathrm{ISCO}}$ and $L_{\text {disk }} / L_{\mathrm{Edd}}$, and thus the disk evolution sequence moves along the diagonal direction rather than vertical direction in Figure 1. If the accretion disk expands linearly with increasing luminosity from $\sim 0.3 L_{\mathrm{Edd}}$, and its evolution sequence is consistent with those of Galactic XRBs, we need to pull down the sequence by increasing the inclination angle $\theta$, or decreasing the value of $\xi_{\text {cor }} f_{\text {col }}^{2}$, and/or spin $a_{*}$. However, it is suggested that the value of $\xi_{\text {cor }} f_{\text {col }}^{2}$ might increase slowly with luminosity (e.g., Davis et al. 2005), though the exact value in super-Eddington state is unknown. The large variation amplitudes for the sinusoidal optical light curves indicated the possibility that the inclination angle is larger than $70^{\circ}$ and smaller than $80^{\circ}$ (Liu et al. 2012). Because the value of $R_{\text {disk }} / R_{\text {ISCO }}$ decreases with decreasing of spin $a_{*}$ and $L_{\text {disk }} / L_{\text {Edd }}$ is independent of $a_{*}$, the disk evolution sequence moves down in the inner disk radius-luminosity plane with a large retrograde spin. Thus, we adopt $a_{*}=-1$ and $\theta=80^{\circ}$, and plot 10 of 14 convergent fittings in the inner disk radiusluminosity plane (Figure 1(b)). In Figure 1, the error of inner disk radius is derived from the error of disk component normalization. On the other hand, we assume that the relative error of the disk bolometric luminosity is equal to that of the $0.3-10.0 \mathrm{keV}$ disk luminosity, which is calculated with the convolution model cflux. All errors are in $90 \%$ confidence level. 
Table 1. BEST-FIT SPECTRAL PARAMETERS OF NGC1313 X-2

\begin{tabular}{|c|c|c|c|c|c|c|c|c|c|}
\hline Obs. Date & Instruments & Exposure & $n_{\mathrm{H}}$ & $k T_{\text {disk }}$ & $N_{\text {disk }}$ & $\Gamma$ & $\operatorname{flux}_{\mathrm{X}}$ & $f l u x_{\mathrm{disk}}$ & $\chi^{2} /$ dof \\
\hline \multicolumn{10}{|c|}{ wabs* $($ diskbb + po $)$} \\
\hline 2000 Oct 17 & $\mathrm{PN}$ & 22.5 & $0.29_{-0.04}^{+0.07}$ & $0.21_{-0.05}^{+0.06}$ & $21.2_{-16.5}^{+82.2}$ & $2.21_{-0.15}^{+0.15}$ & $1.95_{-0.29}^{+0.24}$ & $0.54_{-0.22}^{+0.47}$ & $104.1 / 95$ \\
\hline 2003 Nov 25 & $\mathrm{PN} / \mathrm{M} 1 / \mathrm{M} 2$ & $1.3 / 2.6 / 2.6$ & $0.23_{-0.04}^{+0.05}$ & $\ldots$ & $\ldots$ & $1.75_{-0.12}^{+0.12}$ & $3.16_{-0.23}^{+0.23}$ & $\ldots$ & $101.7 / 88$ \\
\hline 2003 Dec 21 & $\mathrm{PN} / \mathrm{M} 1 / \mathrm{M} 2$ & $7.6 / 10.6 / 10.6$ & $0.47_{-0.07}^{+0.10}$ & $0.11_{-0.01}^{+0.01}$ & $4066_{-1311}^{+12583}$ & $1.90_{-0.06}^{+0.09}$ & $10.00_{-1.29}^{+6.98}$ & $5.58_{-3.15}^{+6.04}$ & $201.9 / 217$ \\
\hline 2003 Dec 23 & $\mathrm{PN} / \mathrm{M} 1 / \mathrm{M} 2$ & $3.3 / 8.9 / 9.2$ & $0.26_{-0.02}^{+0.02}$ & $\ldots{ }^{-0.01}$ & $\ldots{ }^{-1311}$ & $1.70_{-0.05}^{+0.05}$ & $5.62_{-0.25}^{+0.279}$ & $\ldots^{-3.15}$ & $194.1 / 155$ \\
\hline 2003 Dec 25 & $\mathrm{PN} / \mathrm{M} 1 / \mathrm{M} 2$ & $7.0 / 9.0 / 9.1$ & $0.26_{-0.02}^{+0.02}$ & $\ldots$ & $\ldots$ & $2.19_{-0.07}^{+0.07}$ & $2.69_{-0.12}^{+0.13}$ & $\ldots$ & $139.4 / 115$ \\
\hline 2004 Jan 8 & $\mathrm{PN} / \mathrm{M} 1 / \mathrm{M} 2$ & $9.2 / 12.9 / 13.0$ & $0.28_{-0.02}^{+0.02}$ & $\ldots$ & $\ldots$ & $2.36_{-0.07}^{+0.07}$ & $2.45_{-0.11}^{+0.12}$ & $\ldots$ & $138.4 / 131$ \\
\hline 2004 Jan 17 & $\mathrm{PN} / \mathrm{M} 1 / \mathrm{M} 2$ & $5.3 / 8.4 / 8.4$ & $0.27_{-0.03}^{+0.03}$ & $\cdots$ & $\ldots$ & $2.38_{-0.10}^{+0.01}$ & $2.14_{-0.14}^{+0.11}$ & $\ldots$ & $70.0 / 70$ \\
\hline 2004 May 1 & $\mathrm{M} 1 / \mathrm{M} 2$ & $9.2 / 9.8$ & $0.31_{-0.04}^{+0.04}$ & $\cdots$ & $\cdots$ & $2.56_{-0.14}^{+0.15}$ & $2.19_{-0.24}^{+0.32}$ & $\ldots$ & $74.6 / 84$ \\
\hline 2004 Jun 5 & $\mathrm{PN} / \mathrm{M} 1 / \mathrm{M} 2$ & $8.8 / 11.5 / 11.5$ & $0.53_{-0.07}^{+0.08}$ & $0.12_{-0.01}^{+0.01}$ & $4858_{-3186}^{+9136}$ & $1.94_{-0.05}^{+0.06}$ & $12.02_{-2.25}^{+9.85}$ & $6.96_{-3.60}^{+6.61}$ & $290.0 / 280$ \\
\hline 2004 Aug 23 & $\mathrm{PN} / \mathrm{M} 1 / \mathrm{M} 2$ & $10.0 / 14.7 / 14.9$ & $0.22_{-0.05}^{+0.07}$ & $0.26_{-0.07}^{+0.01}$ & $5.78_{-4.3}^{+28.7}$ & $1.82_{-0.31}^{+0.22}$ & $1.51_{-0.10}^{+0.22}$ & $0.42_{-0.11}^{+0.24}$ & $124.3 / 109$ \\
\hline 2004 Nov 23 & $\mathrm{PN} / \mathrm{M} 1 / \mathrm{M} 2$ & $12.4 / 15.5 / 15.5$ & $0.28_{-0.04}^{+0.07}$ & $0.22_{-0.05}^{+0.07}$ & $14.4_{-11.2}^{+9.3}$ & $2.18_{-0.14}^{+0.31}$ & $1.95_{-0.29}^{+0.45}$ & $0.45_{-0.19}^{+0.45}$ & $136.0 / 125$ \\
\hline 2005 Feb 7 & $\mathrm{PN} / \mathrm{M} 1 / \mathrm{M} 2$ & $9.6 / 12.8 / 13.2$ & $0.56_{-0.07}^{+0.07}$ & $0.11_{-0.01}^{+0.01}$ & $12066_{-7203}^{+17429}$ & $1.89_{-0.05}^{+0.06}$ & $21.88_{-10.40}^{+3.83}$ & $10.46_{-4.91}^{+7.96}$ & $395.6 / 292$ \\
\hline 2006 Mar 6 & $\mathrm{PN}$ & 17.4 & $0.53_{-0.09}^{+0.08}$ & $0.11_{-0.01}^{+0.01}$ & $6864_{-4878}^{+12033}$ & $1.99_{-0.07}^{+0.06}$ & $\begin{array}{l}12.30_{-3.79}^{+6.75} \\
\end{array}$ & $6.34_{-3.82}^{+6.45}$ & $241.3 / 195$ \\
\hline 2006 Oct 15 & $\mathrm{PN}$ & 84.0 & $0.52_{-0.03}^{+0.05}$ & $0.11_{-0.01}^{+0.01}$ & $5726_{-1941}^{+5776}$ & $1.98_{-0.02}^{+0.04}$ & $12.30_{-1.83}^{+1.82}$ & $7.08_{-2.15}^{+2.79}$ & $776.0 / 646$ \\
\hline \multicolumn{10}{|c|}{ wabs*simpl*diskbb } \\
\hline 2000 Oct 17 & $\mathrm{PN}$ & 22.5 & $0.26_{-0.05}^{+0.08}$ & $0.21_{-0.05}^{+0.06}$ & $32.3^{+138.3}$ & $2.20_{-0.16}^{+0.14}$ & $1.70_{-0.29}^{+0.59}$ & $0.81_{-0.19}^{+0.45}$ & $103.5 / 95$ \\
\hline 2003 Nov 25 & $\mathrm{PN} / \mathrm{M} 1 / \mathrm{M} 2$ & $1.3 / 2.6 / 2.6$ & $0.60_{-0.18}^{+0.19}$ & $0.09_{-0.01}^{+0.02}$ & $45537^{+493264}$ & $2.08_{-0.19}^{+0.19}$ & $19.95_{-13.64}^{+59.48}$ & $16.56_{-13.28}^{+60.71}$ & $90.6 / 86$ \\
\hline 2003 Dec 21 & $\mathrm{PN} / \mathrm{M} 1 / \mathrm{M} 2$ & $7.6 / 10.6 / 10.6$ & $0.48_{-0.08}^{+0.09}$ & $0.11_{-0.01}^{+0.01}$ & $5196_{-3453}^{+11007}$ & $1.90_{-0.07}^{+0.08}$ & $\begin{array}{l}11.22_{-3.63}^{+6.164} \\
6\end{array}$ & $6.18_{-3.32}^{+\overline{5} .91^{2}}$ & $203.3 / 217$ \\
\hline 2003 Dec 23 & $\mathrm{PN} / \mathrm{M} 1 / \mathrm{M} 2$ & $3.3 / 8.9 / 9.2$ & $0.42_{-0.14}^{+0.11}$ & $0.13_{-0.01}^{+0.01}$ & $1470_{-1225}^{+4851}$ & $1.80_{-0.09}^{+0.09}$ & $8.71_{-2.82}^{+5.74}$ & $3.52_{-2.43}^{+5.31}$ & $190.0 / 153$ \\
\hline 2003 Dec 25 & $\mathrm{PN} / \mathrm{M} 1 / \mathrm{M} 2$ & $7.0 / 9.0 / 9.1$ & $0.40_{-0.09}^{+0.11}$ & $0.12_{-0.02}^{+0.01}$ & $1355_{-1219}^{+5309}$ & $2.27_{-0.12}^{+0.12}$ & $4.57_{-1.94}^{+4.34}$ & $2.33_{-1.64}^{+3.97}$ & $135.4 / 113$ \\
\hline $2004 \operatorname{Jan} 8$ & $\mathrm{PN} / \mathrm{M} 1 / \mathrm{M} 2$ & $9.2 / 12.9 / 13.0$ & $0.28_{-0.02}^{+0.15}$ & $\begin{array}{l}{ }^{-0.02} \\
0.15_{-0.06}^{+0.10}\end{array}$ & $133.2_{-41.2}^{+3037.7}$ & $2.32_{-0.09}^{+0.07}$ & $2.34_{-0.11}^{+2.94}$ & $0.68_{-0.36}^{+2.04}$ & $135.9 / 129$ \\
\hline 2004 Jan 17 & $\mathrm{PN} / \mathrm{M} 1 / \mathrm{M} 2$ & $5.3 / 8.4 / 8.4$ & $0.22_{-0.03}^{+0.11}$ & $0.25_{-0.12}^{+0.14}$ & $12.8^{+172.2}$ & $2.21_{-0.29}^{+0.13}$ & $1.74_{-0.26}^{+0.77}$ & $0.72_{-0.09}^{+0.47}$ & $66.8 / 68$ \\
\hline 2004 May 1 & M1/M2 & $9.2 / 9.8$ & $0.37_{-0.19}^{+0.29}$ & $0.12^{+0.43}$ & $774.3^{+31974.9}$ & $2.60_{-0.18}^{+0.30}$ & $2.75_{-0.98}^{+13.84}$ & $1.22_{-1.21}^{+12.85}$ & $74.2 / 82$ \\
\hline 2004 Jun 5 & $\mathrm{PN} / \mathrm{M} 1 / \mathrm{M} 2$ & $8.8 / 11.5 / 11.5$ & $0.53_{-0.08}^{+0.08}$ & $0.12_{-0.01}^{+0.01}$ & $5585_{-3520}^{+9232}$ & $1.94_{-0.06}^{+0.06}$ & $14.13_{-4.35}^{+6.77}$ & $8.27_{-4.03}^{+6.55}$ & $290.7 / 280$ \\
\hline 2004 Aug 23 & $\mathrm{PN} / \mathrm{M} 1 / \mathrm{M} 2$ & $10.0 / 14.7 / 14.9$ & $0.19_{-0.05}^{+0.08}$ & $0.27_{-0.08}^{+0.08}$ & $7.3_{-5.0}^{+36.7}$ & $1.80_{-0.27}^{+0.23}$ & $1.38_{-0.15}^{+0.36}$ & $0.64_{-0.10}^{+0.21}$ & $124.4 / 109$ \\
\hline 2004 Nov 23 & $\mathrm{PN} / \mathrm{M} 1 / \mathrm{M} 2$ & $12.4 / 15.5 / 15.5$ & $0.26_{-0.06}^{+0.07}$ & $0.21_{-0.04}^{+0.0 \%}$ & $32_{-24.8}^{+143.6}$ & $2.18_{-0.16}^{+0.12}$ & $\begin{array}{l}1.70_{-0.29}^{+0.166} \\
\text {-0.2 }\end{array}$ & $0.78_{-0.17}^{+0.49}$ & $135.0 / 125$ \\
\hline 2005 Feb 7 & $\mathrm{PN} / \mathrm{M} 1 / \mathrm{M} 2$ & $9.6 / 12.8 / 13.2$ & $0.57_{-0.07}^{+0.07}$ & $0.11_{-0.01}^{+0.01}$ & $14015_{-8149}^{+18025}$ & $1.89_{-0.03}^{+0.06}$ & $17.78_{-5.48}^{+8.52}$ & $11.95_{-5.19}^{+8.36}$ & $390.5 / 292$ \\
\hline 2006 Mar 6 & $\mathrm{PN}$ & 17.4 & $0.52_{-0.08}^{+0.09}$ & $0.11_{-0.01}^{+0.01}$ & $7049_{-4459}^{+13354}$ & $1.99_{-0.06}^{+0.05}$ & $12.59_{-4.08}^{+6.91}$ & $6.96_{-3.77}^{+6.64}$ & $241.4 / 195$ \\
\hline 2006 Oct 15 & $\mathrm{PN}$ & 84.0 & $0.52_{-0.04}^{+0.08}$ & $0.11_{-0.01}^{+0.01}$ & $7326_{-2824}^{+4858}$ & $1.99_{-0.03}^{+0.03}$ & $12.88_{-2.41}^{+2.97}$ & $\begin{array}{l}7.77_{-2.18}^{+2.78} \\
\text { - }\end{array}$ & $775.9 / 646$ \\
\hline
\end{tabular}

Note. - Instruments: PN, MOS1(M1) or MOS2(M2); Exposure: clean exposure in units of ksec for the corresponding instrument after background flares excluded; $n_{\mathrm{H}}$ : column density in units of $10^{22} \mathrm{~cm}^{-2}$ along the line of sight; $k T_{\text {disk }}$ : inner disk temperature (in units of keV) of the multicolor disk component, which is unavailable when the disk component is less than $99 \%$ of confidence level; $N_{\text {disk }}$ : normalization of the disk component; $\Gamma$ : power-law photon index; flux $\mathrm{X}$ : $0.3-10 \mathrm{keV}$ flux in units of $10^{-12} \mathrm{erg} \mathrm{cm}^{-2} \mathrm{~s}^{-1} ;$ flux $\mathrm{disk}_{\mathrm{si}}: 0.3-10 \mathrm{keV} \mathrm{disk} \mathrm{flux} \mathrm{in} \mathrm{units} \mathrm{of} 10^{-12}$ erg cm $\mathrm{s}^{-1} ; \chi^{2} / \mathrm{dof}^{2} \chi^{2}$ and degree of freedom for the best-fit model; All errors are in $90 \%$ confidence level. 


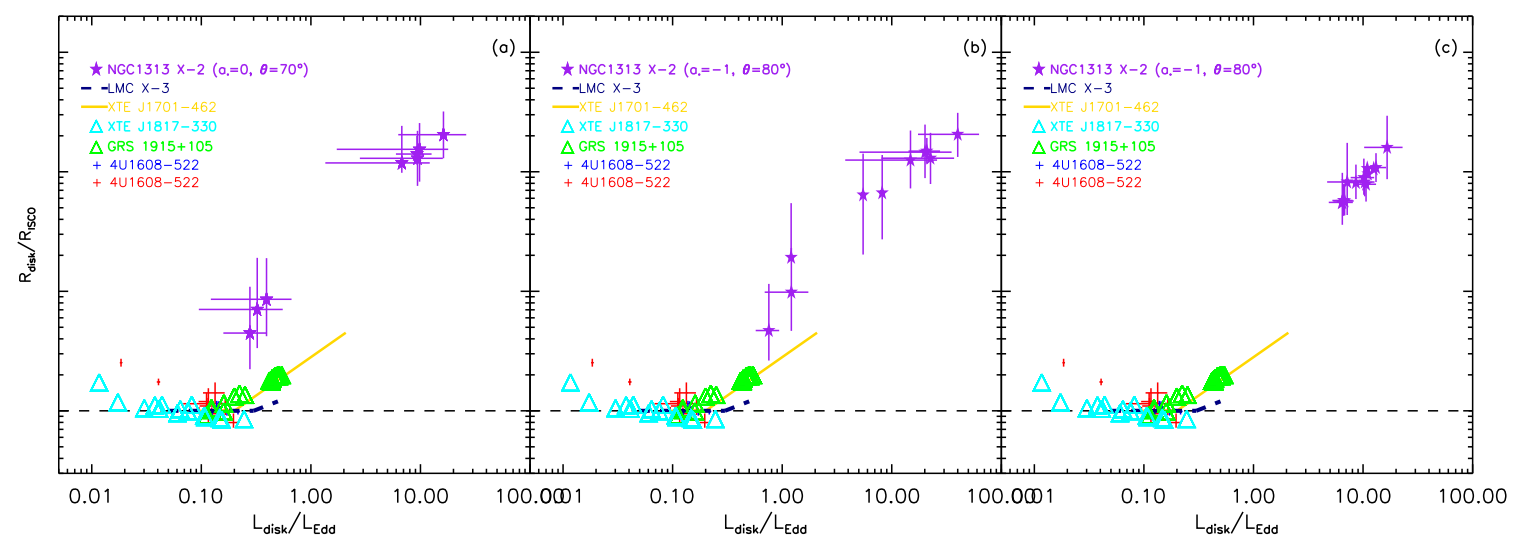

Fig. 1.- Relation between normalized inner disk radius and normalized disk luminosity. NGC1313 X-2 data are shown with the Galactic sources (Paper I) in the inner disk radiusluminosity plane. a) wabs (diskbb+ powerlaw) fit: A Schwarzschild BH with mass of $50 M_{\odot}$, the color correction factor $f_{\text {col }}=1.7$, the correction factor $\xi_{\text {cor }}=0.41$, and an inclination angle $\theta=70^{\circ}$ are adopted here. b) wabs $*$ simpl $*$ diskbb fit: BH spin $a_{*}=-1$ and the inclination angle $\theta=80^{\circ}$. c) the same as b), except that $N_{\mathrm{H}}$ is assumed to be the same for all 14 observations.

Table 2. SPECTRAL FITS WITH THE $t b a b s *(d i s k b b+p o)$ MODEL

\begin{tabular}{|c|c|c|c|c|c|c|c|}
\hline Obs. Date & $n_{\mathrm{H}}$ & $k T_{\text {disk }}$ & $N_{\text {disk }}$ & $\Gamma$ & $\operatorname{flux}_{\mathrm{X}}$ & $f l u x_{\text {disk }}$ & $\chi^{2} /$ dof \\
\hline 2000 Oct 17 & $0.34_{-0.05}^{+0.09}$ & $0.19_{-0.04}^{+0.04}$ & $52.5_{-39}^{+279.9}$ & $2.23^{+0.16}$ & $2.50_{-0.62}^{+0.37}$ & $0.92_{-0.41}^{+0.94}$ & $100.8 / 95$ \\
\hline 2003 Nov 25 & $0.23_{-0.04}^{+0.05}$ & $\ldots$ & $\ldots$ & $1.77_{-0.12}^{+0.14}$ & $3.22_{-0.25}^{+0.62}$ & $\ldots{ }^{-0.41}$ & $100.0 / 88$ \\
\hline 2003 Dec 21 & $\begin{array}{l}0.52_{-0.09}^{+0.09} \\
0\end{array}$ & $0.12_{-0.01}^{+0.01}$ & $4150_{-3045}^{+8716}$ & $1.94_{-0.07}^{+0.07}$ & $10.08_{-2.22}^{+10.31}$ & $6.58_{-3.88}^{+7.04}$ & $199.9 / 217$ \\
\hline 2003 Dec 23 & $0.27_{-0.02}^{+0.02}$ & $\cdots$ & $\cdots$ & $1.72_{-0.05}^{+0.05}$ & $5.70_{-0.21}^{+0.21}$ & $\cdots$ & $192.4 / 155$ \\
\hline 2003 Dec 25 & $0.27_{-0.02}^{+0.02}$ & $\ldots$ & $\ldots$ & $2.23_{-0.07}^{+0.07}$ & $2.76_{-0.14}^{+0.15}$ & $\ldots$ & $140.8 / 115$ \\
\hline 2004 Jan 8 & $0.29_{-0.02}^{+0.02}$ & $\ldots$ & $\ldots$ & $2.40_{-0.07}^{+0.07}$ & $2.54_{-0.14}^{+0.16}$ & $\ldots$ & $142.4 / 131$ \\
\hline 2004 Jan 17 & $0.28_{-0.03}^{+0.03}$ & $\ldots$ & $\ldots$ & $2.41_{-0.10}^{+0.11}$ & $2.24_{-0.18}^{+0.22}$ & $\ldots$ & $71.5 / 70$ \\
\hline 2004 May 1 & $0.32_{-0.02}^{+0.05}$ & $\ldots$ & $\ldots$ & $2.59^{+0.18}$ & $2.31_{-0.28}^{+0.18}$ & $\ldots$ & $75.2 / 84$ \\
\hline 2004 Jun 5 & $0.60_{-0.08}^{+0.08}$ & $0.12_{-0.01}^{+0.01}$ & $5790_{-3891}^{+9788}$ & $1.99_{-0.06}^{+0.13}$ & $16.45_{-4.40}^{+7.78}$ & $10.73_{-5.52}^{+9.14}$ & $282.2 / 280$ \\
\hline 2004 Aug 23 & $0.24_{-0.06}^{+0.09}$ & $0.25_{-0.07}^{+0.01}$ & $8.3_{-6.1}^{+\overline{6 s}_{3}}$ & $1.83_{-0.27}^{+0.24}$ & $1.61_{-0.25}^{+0.07}$ & $0.53_{-0.16}^{+0.532}$ & $124.4 / 109$ \\
\hline 2004 Nov 23 & $0.33_{-0.05}^{+0.08}$ & $0.20_{-0.04}^{+0.05}$ & $35.0_{-27.4}^{+199.0}$ & $2.21_{-0.14}^{+0.14}$ & $2.37_{-0.56}^{+0.15}$ & $0.74_{-0.32}^{+0.80}$ & $135.7 / 125$ \\
\hline 2005 Feb 7 & $0.61_{-0.08}^{+0.08}$ & $0.12_{-0.01}^{+0.01}$ & $10402_{-6470}^{+15678}$ & $1.92_{-0.06}^{+0.06}$ & $19.42_{-6.41}^{+6.50}$ & $12.92_{-6.22}^{+10.24}$ & $392.7 / 292$ \\
\hline 2006 Mar 6 & $\begin{array}{l}0.59_{-0.09}^{+0.09} \\
\end{array}$ & $0.12_{-0.01}^{+0.01}$ & $6035_{-4317}^{+12766}$ & $2.03_{-0.07}^{+0.07}$ & $15.86_{-5.62}^{+7.30}$ & $9.31_{-5.46}^{+9.88}$ & $232.8 / 195$ \\
\hline 2006 Oct 15 & $0.58_{-0.04}^{+0.04}$ & $0.12_{-0.01}^{+0.01}$ & $6234_{-2720}^{+4293}$ & $2.03_{-0.03}^{+0.03}$ & $14.83_{-2.45}^{+2.24}$ & $9.35_{-2.87}^{+3.72}$ & $759.2 / 646$ \\
\hline
\end{tabular}

Note. - All errors are in $90 \%$ confidence level. 
Table 3. SPECTRAL FITS WITH THE wabs $*$ simpl $*$ diskbb MODEL

\begin{tabular}{|c|c|c|c|c|c|c|}
\hline Obs. Date & $n_{\mathrm{H}}$ & $k T_{\text {disk }}$ & $N_{\text {disk }}$ & $\Gamma$ & $\operatorname{flux}_{\mathrm{X}}$ & flux $_{\mathrm{disk}}$ \\
\hline 2000 Oct 17 & $0.45^{+0.02}$ & $0.13^{+0.01}$ & $1093^{+970}$ & $2.41^{+0.11}$ & $4.19^{+0.76}$ & $2.93^{+0.68}$ \\
\hline 2003 Nov 25 & $0.45_{-0.02}^{+0.02}$ & $0.10_{-0.01}^{+0.02}$ & $8462_{-5972}^{+20181}$ & $1.94_{-0.10}^{+0.11}$ & $7.98^{-2.62}$ & $4.72_{-1.51}^{+2.55}$ \\
\hline 2003 Dec 21 & $0.45_{-0.02}^{+0.02}$ & $0.11_{-0.01}^{+0.01}$ & $3895_{-1667}^{+2878}$ & $1.89_{-0.04}^{+0.11}$ & $9.56_{-1.10}^{+1.04}$ & $4.72_{-1.01}^{+1.21}$ \\
\hline 2003 Dec 23 & $0.45_{-0.02}^{+0.02}$ & $0.13_{-0.01}^{+0.01}$ & $2046_{-996}^{+2061}$ & $1.82_{-0.05}^{+0.05}$ & $9.79_{-1.07}^{+1.30}$ & $4.48_{-0.94}^{+1.15}$ \\
\hline 2003 Dec 25 & $0.45_{-0.02}^{+0.02}$ & $0.12_{-0.01}^{+0.01}$ & $2644_{-1280}^{+2854}$ & $2.31_{-0.08}^{+0.08}$ & $6.24_{-0.93}^{+1.15}$ & $3.86_{-0.83}^{+1.03}$ \\
\hline 2004 Jan 8 & $0.45_{-0.02}^{+0.02}$ & $0.12_{-0.01}^{+0.01}$ & $2186_{-1029}^{+2178}$ & $2.46_{-0.08}^{+0.08}$ & $5.32_{-0.79}^{+0.97}$ & $3.29_{-0.70}^{+0.86}$ \\
\hline 2004 Jan 17 & $0.45_{-0.02}^{+0.02}$ & $0.13_{-0.02}^{+0.01}$ & $1018_{-589}^{+2162}$ & $2.43_{-0.14}^{+0.08}$ & $4.57_{-0.74}^{+1.01}$ & $2.92_{-0.63}^{+0.83}$ \\
\hline 2004 May 1 & $0.45_{-0.02}^{+0.02}$ & $0.11_{-0.02}^{+0.03}$ & $2234_{-1609}^{+7844}$ & $2.67_{-0.15}^{+0.15}$ & $4.28_{-0.89}^{+1.21}$ & $2.53_{-0.73}^{+1.01}$ \\
\hline 2004 Jun 5 & $\begin{array}{l}-0.02 \\
0.45_{-0.02}^{+0.02}\end{array}$ & $\begin{array}{l}-0.02 \\
0.12_{-0.01}^{+0.01}\end{array}$ & $2231_{-943}^{+1609}$ & $\begin{array}{l}-0.15 \\
1.89_{-0.04}^{+0.04}\end{array}$ & 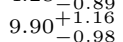 & $\begin{array}{r}{ }^{-0.73} \\
4.18_{-0.88}^{+1.04}\end{array}$ \\
\hline 2004 Aug 23 & $0.45_{-0.02}^{+0.02}$ & $0.13_{-0.01}^{+0.01}$ & $\begin{array}{r}1075_{-459}^{+973} \\
+97\end{array}$ & $2.23_{-0.11}^{+0.04}$ & $4.20_{-0.63}^{+0.78}$ & $3.05_{-0.57}^{+0.88}$ \\
\hline 2004 Nov 23 & $0.45_{-0.02}^{+0.02}$ & $0.13_{-0.01}^{+0.01}$ & $1005_{-422}^{+869}$ & $2.38_{-0.09}^{+0.09}$ & $4.19_{-0.60}^{+0.63}$ & $2.87_{-0.54}^{+0.55}$ \\
\hline 2005 Feb 7 & $0.45_{-0.02}^{+0.02}$ & $0.11_{-0.01}^{+0.01}$ & $3703_{-1491}^{+2428}$ & $\begin{array}{l}1.81_{-0.03}^{+0.03} \\
\end{array}$ & $9.87_{-1.04}^{+1.22}$ & $4.50_{-0.95}^{+1.12}$ \\
\hline 2006 Mar 6 & $0.45_{-0.02}^{+0.02}$ & $0.11_{-0.01}^{+0.01}$ & $3749_{-1844}^{+3824}$ & $1.94_{-0.04}^{+0.04}$ & $9.14_{-0.97}^{+1.17}$ & $3.72_{-0.87}^{+1.04}$ \\
\hline 2006 Oct 15 & $0.45_{-0.02}^{+0.02}$ & $0.12_{-0.01}^{+0.01}$ & $3046_{-947}^{+1316}$ & $1.94_{-0.02}^{+0.02}$ & $8.93_{-0.91}^{+1.06}$ & $4.10_{-0.84}^{+0.97}$ \\
\hline
\end{tabular}

Note. - All 14 observations are fitted simultaneously with the same value of $N_{\mathrm{H}}$, and $\chi^{2} /$ dof $=3043.4 / 2603$ is obtained. All errors are in 90\% confidence level.

Table 4. SPECTRAL FITS WITH THE wabs $*$ diskir MODEL

\begin{tabular}{|c|c|c|c|c|c|c|c|c|c|}
\hline Obs. Date & $n_{\mathrm{H}}$ & $k T_{\text {disk }}$ & $\Gamma$ & $k T_{\mathrm{e}}$ & $L c / L d$ & rirr & $N_{\text {disk }}$ & $f^{\prime l u x_{\mathrm{X}}}$ & $\chi^{2} /$ dof \\
\hline 2000 Oct 17 & $0.23_{-0.07}^{+0.03}$ & $0.18_{-0.03}^{+0.11}$ & $2.20_{-0.12}^{+0.12}$ & $930.6-929.6$ & $2.89_{-2.34}$ & $1.75_{-0.63}$ & $24.7_{-20.5}^{+213.9}$ & $1.53_{-0.49}^{+0.58}$ & $102.9 / 93$ \\
\hline 2003 Nov 25 & $0.17_{-0.13}^{+0.12}$ & $0.23_{-0.07}^{+1.27}$ & $2.06_{-0.53}^{+0.86}$ & $25.9-24.5$ & $4.52_{-4.50}$ & $1.02_{-0.01}^{+0.07}$ & $14.0^{-20.5}$ & $\begin{array}{r}2.86_{-0.87}^{+0.49} \\
2.87\end{array}$ & $89.6 / 84$ \\
\hline 2003 Dec 21 & $0.28_{-0.09}^{+0.02}$ & $0.11_{-0.04}^{+0.15}$ & $1.72_{-0.05}^{+0.06}$ & $1.83_{-0.19}^{+0.16}$ & $10.0_{-9.29}$ & $2.01_{-0.82}$ & $166.0_{-80.5}^{+5310.3}$ & $\begin{array}{l}4.94_{-0.48}^{+2.84} \\
\end{array}$ & $181.6 / 215$ \\
\hline 2003 Dec 23 & $0.21_{-0.01}^{+0.01}$ & $0.08_{-0.01}^{+0.01}$ & $1.54_{-0.02}^{+0.02}$ & $1.41_{-0.08}^{+0.01}$ & $2.84_{-0.53}^{+0.67}$ & $1.00_{-0.01}^{+0.01}$ & $2249_{-68}^{+2772}$ & $4.81_{-0.17}^{+0.98}$ & $165.6 / 151$ \\
\hline 2003 Dec 25 & $0.39_{-0.17}^{+0.11}$ & $0.12_{-0.03}^{+0.19}$ & $2.14_{-0.17}^{+0.18}$ & $2.84_{-1.21}$ & $0.40_{-0.24}$ & $1.02-0.02$ & 1503 & 4.24 & $134.1 / 111$ \\
\hline $2004 \operatorname{Jan} 8$ & $0.23_{-0.12}^{+0.04}$ & $0.16_{-0.07}^{+0.25}$ & $2.21_{-0.21}^{+0.15}$ & $3.13-1.60$ & $8.12_{-4.30}$ & $3.27-1.50$ & 15.8 & $1.98_{-0.47}^{+2.42}$ & $135.3 / 127$ \\
\hline 2004 Jan 17 & $0.22_{-0.05}^{+0.07}$ & $0.31_{-0.06}^{+0.08}$ & $1.00^{+0.38}$ & $6.38-1.13$ & $4.69_{-2.02}^{+1.02}$ & $1.02_{-0.01}^{+0.02}$ & $5.97_{-4.37}^{+13.62}$ & $1.92_{-0.27}^{+0.41}$ & $61.1 / 66$ \\
\hline 2004 May 1 & $0.22_{-0.07}^{+0.02}$ & $0.33_{-0.15}^{+0.15}$ & $7.34_{-6.21}$ & 1.00 & $0.49_{-0.10}^{+0.43}$ & $1.00_{-0.01}^{+0.01}$ & $4.23_{-4.23}^{+11.91}$ & $1.32_{-0.21}^{+0.29}$ & $67.9 / 80$ \\
\hline 2004 Jun 5 & $0.23_{-0.03}^{+0.05}$ & $0.40_{-0.04}^{+0.04}$ & $2.78_{-0.48}^{+0.53}$ & 767.6 & $2.28_{-0.59}^{+0.78}$ & $1.01_{-0.01}^{+0.01}$ & $3.56_{-1.86}^{+7.06}$ & $5.27_{-0.27}^{+0.37}$ & $230.6 / 278$ \\
\hline 2004 Aug 23 & $0.24_{-0.10}^{+0.10}$ & $0.22_{-0.06}^{+0.04}$ & $\begin{array}{l}1.55_{-0.28}^{+0.48} \\
\end{array}$ & $8.84_{-6.40}^{+9.86}$ & $1.58_{-0.81}^{+1.08}$ & $1.01_{-0.01}^{+0.06}$ & $24.4^{-1.00}$ & $\begin{array}{l}1.63_{-0.30}^{+0.79} \\
\end{array}$ & $120.8 / 107$ \\
\hline 2004 Nov 23 & $0.22_{-0.06}^{+0.07}$ & $0.20_{-0.12}^{+0.13}$ & $2.17_{-0.18}^{+0.10}$ & $266.6_{-263.1}$ & $4.31_{-3.84}$ & $2.36_{-1.22}^{+1.17}$ & $11.5_{-9.8}^{+240.6}$ & $1.45_{-0.23}^{+1.00}$ & $135.1 / 123$ \\
\hline 2005 Feb 7 & $0.23_{-0.02}^{+0.07}$ & $0.28_{-0.08}^{+0.15}$ & $1.61_{-0.07}^{+0.32}$ & $1.69_{-0.22}^{+0.54}$ & $2.60_{-0.51}^{+0.91}$ & $1.02_{-0.01}^{+0.04}$ & $11.6_{-10.0}^{+32.8}$ & $\begin{array}{l}4.89_{-0.38}^{+0.78} \\
\end{array}$ & $330.1 / 290$ \\
\hline 2006 Mar 6 & $0.25_{-0.01}^{+0.01}$ & $0.08_{-0.01}^{+0.01}$ & $1.59_{-0.02}^{+0.02}$ & $1.47_{-0.08}^{+0.01}$ & $2.30_{-0.31}^{+0.31}$ & $1.00_{-0.01}^{+0.01}$ & $2138_{-33}^{+2141}$ & $4.86_{-0.07}^{+0.08}$ & $178.8 / 193$ \\
\hline 2006 Oct 15 & $0.25_{-0.02}^{+0.03}$ & $0.27_{-0.04}^{+0.07}$ & $1.84_{-0.13}^{+0.15}$ & $2.12_{-0.31}^{+0.52}$ & $1.95_{-0.17}^{+0.33}$ & $1.01_{-0.01}^{+0.01}$ & $14.5_{-9.2}^{+18.0}$ & $4.54_{-0.22}^{+0.33}$ & $638.4 / 644$ \\
\hline
\end{tabular}

Note. $-k T_{\text {disk }}$ : inner disk temperature (in units of $\mathrm{keV}$ ); $\Gamma$ : power-law photon index; $k T_{\mathrm{e}}$ : electron temperature; $L c / L d$ : ratio of luminosity in the Compton tail to that of the unilluminated disk; rirr: radius of the Compton illuminated disk in terms of the inner disk radius; $N_{\text {disk }}$ normalization of the disk component; All errors are in $90 \%$ confidence level. 


\section{Temporal Variability}

We extract the light curves from PN data with bin sizes of $200 \mathrm{~s}$ and $1 \mathrm{~s}$ from the same regions as extracting the spectra. We use the task epiclccorr to correct for good time intervals, dead time, and for background subtraction. We first search for possible periodic modulations in its entire $0.2-10.0 \mathrm{keV}$ light curve, using the ftool efsearch with a $200 \mathrm{~s}$ bin-size and a period resolution of 0.02 day. Figure 2 shows the result of chi-squared test over a range of trial periods, i.e., $\chi^{2}$ versus period, in which the red arrow marks the previously suggested orbital period of $P=6.12$ days (Liu et al. 2009). There is no evidence of clear orbital modulation. This can be interpreted as that the intrinsic X-ray spectral evolution and/or flux variations dominate over the orbital modulation during the $\sim$ six years of observation.

The power density spectrum (PSD) is constructed with the continuous exposure $1 \mathrm{~s}$ bin size light curves of $0.2-10.0 \mathrm{keV}$ for each observation. The PSDs can be fitted with a constant power density model indicating the Poisson noise level for all but one observations. The PSD exhibits the red-noise behavior at low frequencies $\left(\sim 10^{-3}-10^{-4} \mathrm{~Hz}\right)$ for the observation of 15 October 2006 (see figure 1 in Heil et al. 2009).

NGC1313 X-2 was observed on 15 October 2006 with a long exposure of 123 ks. After rejecting the data contaminated by the low amplitude flaring particle background, we show the light curves of $0.2-0.8,0.8-2.0,2.0-10.0,0.2-10.0 \mathrm{keV}$, and the hardness ratio between $0.2-0.8 \mathrm{keV}$ and $2.0-10.0 \mathrm{keV}$ in Figure 3. The count rates of $0.2-10.0$ and $2.0-10.0 \mathrm{keV}$ change by a factor of 2 on timescales of $2 \times 10^{4} \mathrm{~s}$, but the soft band emission is remarkably steady. We calculate the fractional variability amplitude $F_{\text {var }}$ with $200 \mathrm{~s}$ bin size light curves to quantify the variability,

$$
F_{\mathrm{var}}=\sqrt{\frac{S^{2}-\overline{\sigma^{2}}}{\bar{x}^{2}}},
$$

where $S^{2}, \sigma^{2}$, and $\bar{x}^{2}$ are the variance, mean error squared, and mean count rate, respectively. The fractional variability amplitude increases with energy, $F_{\text {var }}=4.8 \pm 7.2 \%, 5.5 \pm 1.7 \%$, and $10.2 \pm 1.7 \%$ for $0.2-0.8,0.8-2.0$, and $2.0-10.0 \mathrm{keV}$, respectively.

\section{Discussion}

\subsection{Truncated thin disk}

NGC1313 X-2 provides a textbook of ULX spectrum (Soria 2007; Stobbart et al. 2006). The cool disk component and the large inner disk radius detected in the source have several interpretations (Feng \& Soria 2011). An obvious solution is an IMBH accreting at sub- 


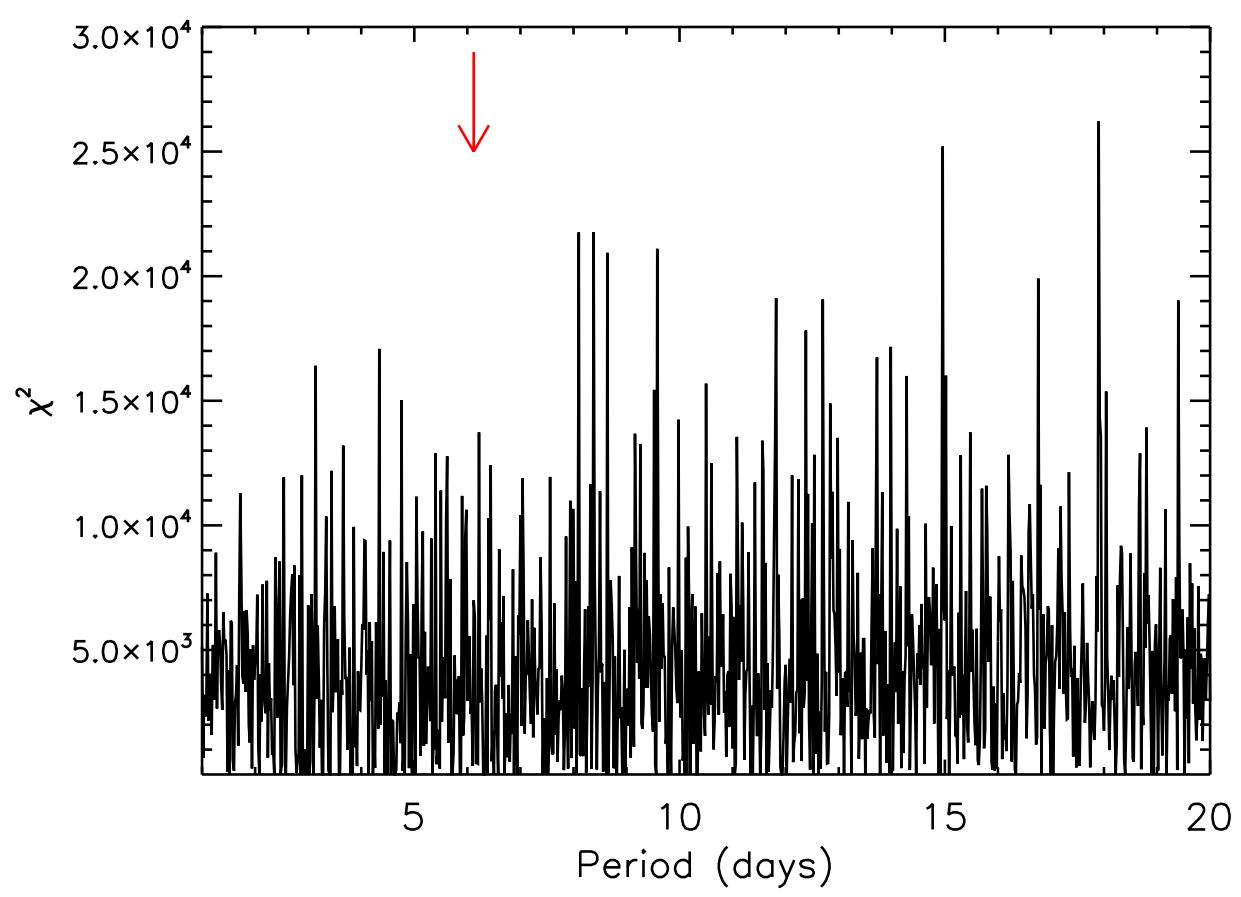

Fig. 2. - $\chi^{2}$ versus trial period generated by efsearch, and the red arrow indicates the reported orbital period of $P=6.12$ days (Liu et al. 2009). 


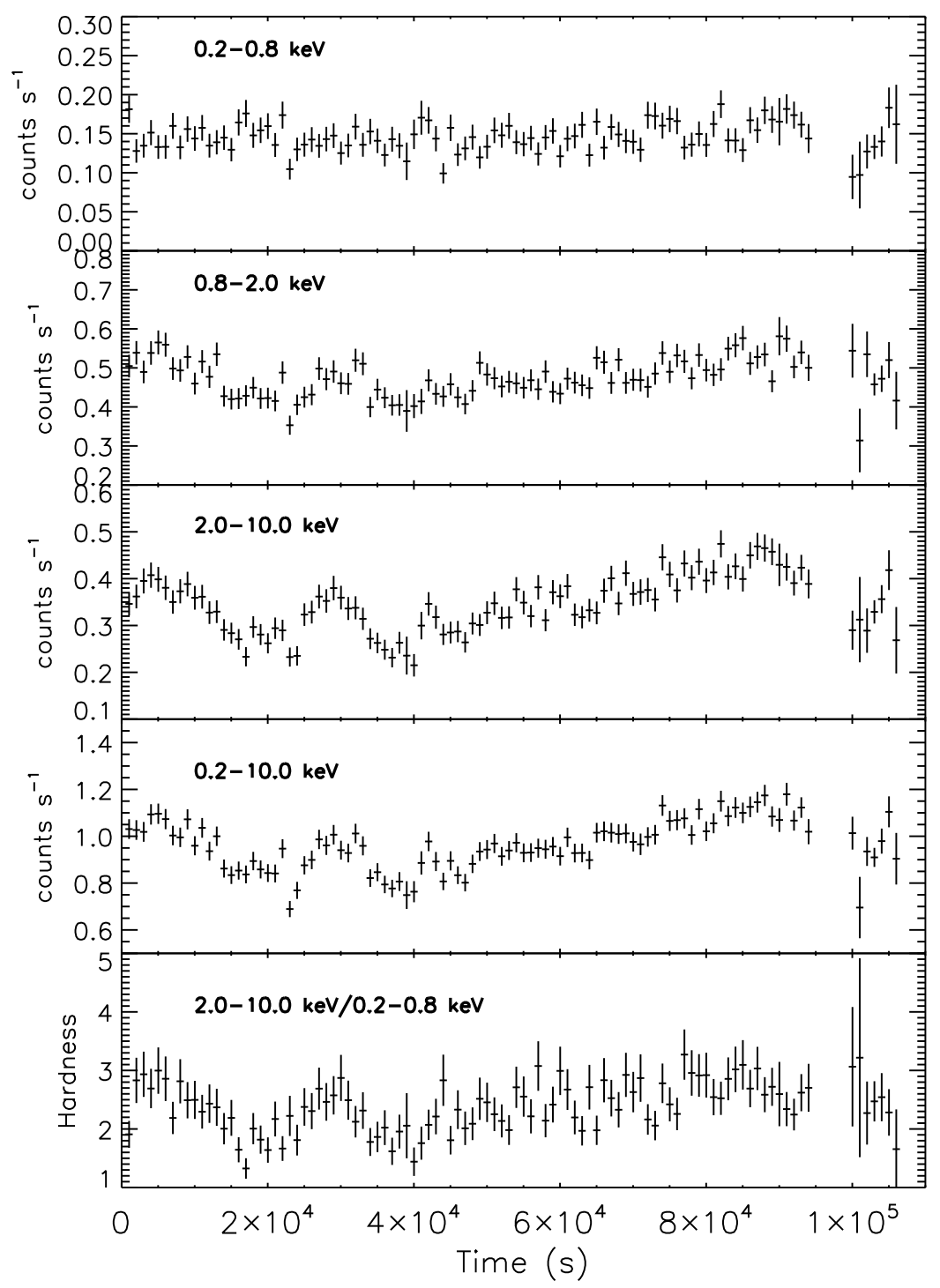

Fig. 3.- Panels from top to bottom show the $1000 \mathrm{~s}$ binned background subtracted light curves in $0.2-0.8,0.8-2.0,2.0-10.0,0.2-10.0 \mathrm{keV}$, and the temporal evolution of the 2.0-10.0 to $0.2-0.8 \mathrm{keV}$ hardness ratios, respectively. 
Eddington rates, and the accretion disk extending to ISCO. However, Feng \& Kaaret (2007) found that the spectral evolution of NGC1313 X-2 is inconsistent with $L_{\text {disk }} \propto T_{\text {disk }}^{4}$ law expected for the standard accretion disk in IMBH. In addition, more and more optical observations (e.g. the ESO VLT and HST data) suggest a MXB in the NGC1313 X-2 (Grisé et al. 2008). Although the luminosity is model-dependent for a given observation, it can hardly vary by an order of magnitude. Assuming the mass of the $\mathrm{BH}$ is $\sim 50 M_{\odot}$ (Zampieri et al. 2004; Liu et al. 2012), the conservatively estimated X-ray luminosity $\sim 10^{40} \mathrm{erg} / \mathrm{s}$ points to a super-critical accretion.

However, there is still no mature theory that can explain all X-ray properties of supercritical accreting sources to date. At high luminosity, the standard disk assumptions break down due to the effect of radiation pressure (Shakura \& Sunyaev 1973). It is still unclear whether the accretion disk is geometrically thin (Begelman 2002; Gu 2012), and whether it is truncated at a large distance from the ISCO in super-Eddington luminosity (Soria 2007). McClintock et al. (2006) argued that the inner disk edge is still located at ISCO, but its emission is shaded by the outer disk when the luminosity goes higher and the disk becomes thicker. Thus the fitting inner disk is not the true value but just the larger radius at which the emission is not blocked by the outer disk. The above effect of model depends on the viewing angle, and it is hard to explain why the different sources give the same evolution pattern at high luminosity. Besides, $\mathrm{Gu}$ (2012) investigated the vertical structure of accretion disks in spherical coordinates and showed that the energy advection is significant even for slightly sub-Eddington accretion disks, and therefore argued that the non-negligible advection may help to understand why the standard disk model is inaccurate above 0.3 Eddington luminosity.

Alternatively, Poutanen et al. (2007) suggested that the cool disk component arose from the spherization radius of a super-critical disk. The model was successfully used to explain several data of ULXs and the anti-correlation between luminosity and temperature (Poutanen et al. 2007). They also predicted that the spherization radius moves out and becomes cooler with increasing luminosity. However, the observed trend is too steep for the predicted relation of this model (Kajava \& Poutanen 2009). Moreover, the luminosity is insensitive to the accretion rate when the source reaches and exceeds the Eddington luminosity, $L \sim L_{\mathrm{Edd}}(1+0.6 \ln \dot{m}$ ) (where $\dot{m}$ is the accretion rate in Eddington units). Thus, this model cannot produce luminosity larger than 10 times of Eddington luminosity unless the beaming effect is taken into account, and the radiative efficiency is less than 0.01 when the luminosity exceeds 3 times of Eddington luminosity (e.g. in NGC1313 X-2).

Begelman (2002) put forward another plausible thin disk model that the radiation pressure dominated accretion disk has strong density inhomogeneities on scales much smaller 
than the disk scale height due to the development of photon-bubble instabilities. The escaping flux from such disk could exceed $L_{\text {Edd }}$ by a factor of up to $10-100$, depending on the $\mathrm{BH}$ mass.

In the specified bright states, the new-found NS XRB, XTE J1701-462, draws the same track with BH XRB, GRS 1915+105, in the inner disk radius-luminosity plane (Paper I; Neilsen et al. 2011). In contrast to the behavior of the inner disk radius, the NS surface emission area maintained its small and nearly constant size. All these information implied that the accretion disk is geometrically thin, and moves out when the source is brighter than $0.3 L_{\text {Edd }}$ (see Paper I for a detail discussion). Besides the X-ray spectral evolution, the lack of short-time variability in the thermal component $(0.2-0.8 \mathrm{keV}$ band emission, Figure 3$)$ implies that the disk is truncated at a large distance from the central BH. Investigating the HST archive data, Tao et al. (2011) also suggested that the accretion disk in NGC1313 $\mathrm{X}-2$ is geometrically thin, and the optical emission was mainly due to its disk irradiation. Therefore, our results are in favor of a truncated thin disk scenario.

In the low/hard spectral state, the thin disk recedes from ISCO, and is irradiated by the Compton tail. This can change the inner disk temperature structure from that expected from an unilluminated disk in the limit where the ratio of luminosity in the tail to that in the disk, $L_{\mathrm{c}} / L_{\mathrm{d}} \gg 1$. The irradiated inner disk and Compton tail can also illuminate the rest of the disk, and a fraction $\left(f_{\text {out }}\right)$ of the bolometric flux is thermalized to the local blackbody temperature at each radius. This reprocessed flux generally dominates the optical and UV bandpass of low-mass X-ray binaries (Gierliński et al. 2008). Now the irradiated inner and outer disk model has been developed to take account of these effects (diskir in XSPEC).

Assuming that the accretion disk in the super-Eddington state has the same geometry as those in the low/hard state, we fit the spectra with diskir model. Because of lacking of $\mathrm{UV} /$ optical data to cover the outer disk information, we fix $f_{\text {out }}$ (fraction of bolometric flux which is thermalized in the outer disk) and logrout (the outer disk radius in terms of the inner disk radius) to the default values. However the XMM-Newton observing window (0.3-10.0 $\mathrm{keV}$ ) is too limited to constrain the parameters of diskir model (e.g., electron temperature and $L_{\mathrm{c}} / L_{\mathrm{d}}$, Table 4$)$. Despite both $R_{\text {disk }}$ and luminosity are systematically lower than those in Table 3, the results indicate that $R_{\text {disk }}$ is tens of ISCO, and the luminosity is $\sim 10^{40} \mathrm{erg} / \mathrm{s}$, which are still not in conflict with our conclusion.

However we cannot obtain reliable information on the non-thermal emission, and can hardly extrapolate its bolometric luminosity with the XMM-Newton limited observing window $(0.3-10.0 \mathrm{keV})$. The $0.3-10.0 \mathrm{keV}$ disk flux is slightly higher than the $0.3-10.0 \mathrm{keV}$ flux of non-thermal emission for $\operatorname{simpl}^{*} d i s k b b$, and the bolometric luminosity of disk emission is a few times of the non-thermal emission $0.3-10.0 \mathrm{keV}$ luminosity. As representative values, we 
adopt $R_{\text {disk }} \sim 50 R_{\mathrm{ISCO}}$ (or $200 R_{\mathrm{S}}$, where $R_{\mathrm{S}}$ is Schwarzschild radius), $L_{\text {total }} \sim L_{\text {disk }} \sim 10^{40}$ $\mathrm{erg} / \mathrm{s}$ (Figure 1 ), and arrive at an accretion rate $\dot{M} \sim \frac{2 L_{\mathrm{total}} R_{\mathrm{disk}}}{G M_{\mathrm{BH}}} \sim 1 \times 10^{-4} M_{\odot} / \mathrm{yr}$, and the

radiative efficiency $\eta_{\text {total }} \sim \frac{G M_{\mathrm{BH}}}{R_{\mathrm{disk}}} / c^{2} \sim 0.003$. Note that the radiative efficiency would be even lower, and accretion rate would be even larger when taking the outflow into account.

The response of the cold material (i.e. an accretion disk) to irradiation by hard Xrays, may produce observable Fe emission lines, which have been frequently detected in both AGNs and XRBs (Miller 2007; Gilfanov 2010). The disk ionization state is dependent on the geometry of the accretion disk. Compared with the thick disk, the thin disk would have higher number density and thus lower ionization parameter. If the accretion disk is truncated at a large radius, we could expect the Fe lines with Doppler shifts but no (or small) gravitational red-shifts.

\subsection{Ultra-fast outflow}

There are two possible views on the way the accretion proceeds between $R_{\text {disk }}$ and $R_{\mathrm{ISCO}}: 1$, The accretion matter reaches the $\mathrm{BH}$, but most of the gravitational energy is released as the photons, which are trapped in the flow and finally advected into the $\mathrm{BH}$ (e.g. Abramowicz et al. 1988); 2, Most of gas is blown away by the radiation pressure, and the accretion rate decreases with the disk radius (e.g. Begelman 2002; Gu \& Lu 2007). The most of power released in the region between $R_{\text {disk }}\left(\sim 50 R_{\mathrm{ISCO}}\right)$ and $R_{\mathrm{ISCO}}$ is eventually radiated as non-thermal power-law photons. In both advection and outflow models, the radiative efficiency of the non-thermal component in the inner region is significantly lower than the efficiency of thermal emission in a standard disk (Soria 2007). In other words, $\eta_{\text {total }} / \eta_{\text {disk }} \sim L_{\text {total }} / L_{\text {disk }}<F$ (Soria 2007), where $F=R_{\text {disk }} / R_{\text {ISCO }} \sim 50$ for NGC1313 X-2.

The emission of non-thermal component increases with the luminosity when the source is close to its Eddington luminosity. The power-law emission of GRS 1915+105 and XTE J1701-462 in Figure 1 contribute less than 25\% of total X-ray flux (McClintock et al. 2006; Paper I). Unlike the cases in canonical low/hard and high/soft states in XRBs, ULXs present no clear gaps, but a broad continuous distribution in photon index $(\Gamma \sim 1.0-3.0$, Feng \& Soria 2011). In NGC1313 X-2, the photon index does not correlate with the disk flux, nor the total flux (Tables 1 and 2). On the whole the non-thermal component contributes a larger fraction of accretion emission comparing with the bright XRBs, but the disk emission still dominates over the non-thermal emission in NGC 1313 X-2 (Section 4.1).

The strong radiatively driven outflows are produced in most highly super-critical accretion models (e.g. Okuda et al. 2005; Begelman et al. 2006; Ohsuga \& Mineshige 2011), and 
the power of outflows is approximately equal to radiation. If the outflow is launched at the inner disk radius $\sim R_{\text {disk }}$ (or the transition radius), the speed of wind is possibly close to the escape velocity, that is, mildly relativistic velocity from $R_{\text {disk }} \sim 200 R_{\mathrm{S}}$ (Figure 1 ). The ultra-fast outflows (UFOs) have been detected in both AGNs and Galactic XRBs (Marshall et al. 2002; Crenshaw \& Kraemer 2012; Tombesi et al. 2012). Recently, King et al. (2012) found the fastest disk wind ( $v_{\text {wind }} \sim 0.03 \mathrm{c}, \mathrm{c}$ is the light speed) in the Galactic source, IGR J17901-3624, with the help of the Chandra X-ray mission.

However, there is still no evidence of atomic iron features that could be associated with massive outflowing material found in ULXs (Walton et al. 2012). Note that the ionized nebulae are revealed not only in many other ULXs (Kaaret et al. 2004; Abolmasov et al. 2007; Moon et al. 2011), but also a subset of XRBs at very high mass accretion rates (Cooke et al. 2008; Pakull et al. 2010), e.g. W50 nebula around SS433 (Fabrika 2004; Goodall et al. 2011). The Doppler-shifted Fe lines together with many other emission lines originating from the high temperature plasma in the conical jets were also detected in SS433 (e.g., Marshall et al. 2002). However, there is no evidence of relativistic jets found in NGC1313 X-2. In addition, the mechanism that UFOs up-scatter the disk seed photons producing the observed non-thermal emissions, has been intensively studied in literature (e.g. Kajava \& Poutanen 2009; Soria 2011). The observed variability shown in 2.0-10.0 keV light curve (Figure 3) can be naturally ascribed to variations of UFOs.

The disappearance of UFOs can be interpreted as UFOs fully ionized or out of our viewing angle. For a toy model, suppose that UFOs are launched from the transition radius $R_{\text {disk }}$ as a sphere expanding with the velocity $v_{\text {UFOs }}$, being the same order of magnitude as the escape velocity at $R_{\text {disk }}, v_{\mathrm{UFOs}} \sim \sqrt{2 G M / R_{\text {disk }}}$. The gas density at the radius of $R$ can be given by,

$$
n_{\mathrm{UFOs}} \sim \frac{\dot{M}_{\mathrm{UFOs}}}{4 \pi R^{2} v_{\mathrm{UFOs}} m_{\mathrm{H}}},
$$

where $m_{\mathrm{H}}$ is the mass of hydrogen atom (or proton) and the mass outflow rate, $\dot{M}_{\mathrm{UFOs}}<$ $\dot{M} \sim 10^{22} \mathrm{~g} / \mathrm{s}$. In this case, the absorber ionisation parameter $\xi_{\text {ion }}$ will be directly related to the source luminosity $L_{\text {total }}$ and $R_{\text {disk }}: \xi_{\text {ion }}>\frac{L_{\text {total }}}{n_{\mathrm{UFOs}} R^{2}}$. Plugging in the typical values, $R_{\text {disk }} \sim 200 R_{\mathrm{S}}, L_{\text {total }} \sim 10^{40} \mathrm{erg} / \mathrm{s}$ (Figure 1 ), the expected ionization parameter $\xi_{\text {ion }}$ is larger than $10^{4.7}$ ergs $\mathrm{cm} \mathrm{s}^{-1}$. That is, UFOs are fully ionized, a fact which accounts for absence of the absorption line (Reynolds \& Nowak 2003).

However, the real geometry of UFOs could be more complicated than the above toy model. If UFOs are clumpy, the localized gas density would be sufficiently high to produce a weak and relatively broad Fe K absorption line (Neilsen et al. 2012), which can be tested with the next generation X-ray mission, e.g. Astro- $H$. Another possibility is that the UFOs 
is anisotropic with small covering factor, and the absorption feature appears only when UFOs occur in our line of sight (Walton et al. 2012). In some rarely violent accretion systems, the mass loss rate from outflows are much larger than the inflow rate derived from the accretion disk (e.g. Pakull et al. 2010; Punsly 2011; Ponti et al. 2012). However, it is unlikely that the companions in ULXs can supply so much accretion matter for a long time (next subsection). The power and low energy conversion efficiency of radiatively driven outflows are probably close to those of X-ray radiation $(\sim 0.003)$ when sources are in superEddington luminosity. That is, the total energy efficiency is less than 2 times of radiative efficiency $(<0.003 \times 2 \sim 0.006)$.

\subsection{Short-lived ionized bubble nebula}

The observations of ESO VLT, HST, and SUBARU reveal that the NGC1313 X-2 is embedded in a huge ionized nebula with a size of $\approx 200 \times 400 \mathrm{pc}$, expanding at $v_{\text {bub }} \approx 100$ $\mathrm{km} \mathrm{s}^{-1}$, which is the highly supersonic expansion speed (e.g. Ramsey et al. 2006). Pakull et al. (2006) argued that the bubble is shock-excited with possible contribution due to photoionization. If the bubble is inflated by a continuous outflow from the central X-ray source, its age $\left(\tau_{\text {bub }} \sim \frac{100 \mathrm{pc}}{100 \mathrm{~km} / \mathrm{s}} \sim 10^{6} \mathrm{yr}\right)$ should be the same as the lifetime of the X-ray active phase $\tau_{\text {acc }}$,

$$
\tau_{\mathrm{acc}} \sim \frac{M_{\mathrm{acc}}}{\dot{M}} \sim \frac{\eta m_{\mathrm{acc}} c^{2}}{2 L_{\mathrm{disk}}}
$$

where $m_{\text {acc }}$ is the total mass transferred from the companion to the $\mathrm{BH}$ during the entire accretion lifetime, and it should be less than the mass of the companion star, $M_{\text {acc }} \sim 1-$ $10 M_{\odot}<M_{\mathrm{MS}}$ (Zampieri et al. 2004; Liu et al. 2012). Thus, a conservative estimate of the accretion age is $\tau_{\text {acc }} \sim 10^{4}-10^{5} \mathrm{yr}$. Taking the outflow into account, the radiative efficiency would be even lower and $\tau_{\text {acc }}$ even shorter. We would like to emphasize that NGC1313 X-2 can emit $10^{40} \mathrm{erg} / \mathrm{s}$ as long as $10^{6} \mathrm{yr}$ only when it accretes $10 M_{\odot}$ with a high radiative efficiency $(\eta \sim 0.02)$.

\subsubsection{Model I: Radiatively driven outflow}

We first investigate the possibility that NGC1313 X-2 is a transient with the duty cycle of activity $\sim \frac{10^{4-5} \mathrm{yr}}{10^{6} \mathrm{yr}}$ and the bubble nebula is resulted from the radiatively driven outflow during the different $\mathrm{X}$-ray active phases. In this scenario, only the earliest $\left(\sim 10^{6} \mathrm{yr}\right.$ ago $)$ outflows are arriving at $\sim 100 \mathrm{pc}$ from the central source with $v_{\text {bub }} \sim 100 \mathrm{~km} \mathrm{~s}^{-1}$ and exciting the outmost part of the bubble nebula; the later outflows have only reached shorter distances 
from the central source and are ionizing the inner part of the bubble nebula. During the propagation in the nebula, the purported outflows are decelerated because of interaction with ISM. Here, we assume that the power of the radiatively driven outflow is $\sim 10^{40} \mathrm{erg} / \mathrm{s}$, which can be transferred $100 \%$ from the outflow to the bubble. Since the mechanical energy stored in the bubble nebula is $E_{\text {bub }} \sim 10^{52} \times\left(\frac{n}{1.0 \mathrm{~cm}^{-3}}\right) \times\left(\frac{R_{\mathrm{bub}}}{100 \mathrm{pc}}\right)^{3} \times\left(\frac{v_{\mathrm{bub}}}{100 \mathrm{~km} / \mathrm{s}}\right)^{2} \mathrm{erg}$, the source should be active no less than $10^{4} \mathrm{yr}\left(\tau_{\text {act }} \geq 10^{4} \mathrm{yr}\right)$ and accrete $\geq 1 M_{\odot}$ to inflate the whole nebula for each duty cycle (or at least the earliest outburst), where $n$ is the number density of the bubble nebula. Otherwise, the earliest outflows should have been decelerated to a velocity lower than the observed $\sim 100 \mathrm{~km} \mathrm{~s}^{-1}$, before reaching a distance of $\sim 100 \mathrm{pc}$ from the central X-ray source. Therefore the source can only be active for a few cycles, that is, the upper limit to the number of cycles is $\sim \frac{\tau_{\text {acc }}}{\tau_{\text {act }}}$. The recombination cooling time scale is $\tau_{\text {cool }} \sim \frac{n}{\alpha_{\mathrm{H}} n^{2}} \sim \frac{1}{\alpha_{\mathrm{H}} n} \sim 10^{5} \times\left(\frac{1.0 \mathrm{~cm}^{-3}}{n}\right)$ yr, where $\alpha_{\mathrm{H}} \sim 3 \times 10^{-13} \mathrm{~cm}^{3} \mathrm{~s}^{-1}$ is the recombination coefficient (Cen \& Haimen 2000). It means that the observed ionized bubble nebula records the last $\tau_{\text {cool }} \sim 10^{5}$ yr ionized path of the outflows generated in different active phases. In other words, the different parts of the bubble nebula are resulted from the outflows produced in different epochs, and the lower limit to the number of duty cycles is $\frac{R_{\text {bub }}}{v_{\text {bub }}\left(\tau_{\text {cool }}+\tau_{\text {act }}\right)}$. This model requires a high radiative efficiency and a high energy transfer efficiency between the radiatively driven outflow and ISM. The number density of ISM also plays a key role in this model: The low-density ISM can be easily accelerated and has a long recombination cooling time, or vice versa. Note that, the upper limit to the number of cycles is close to (or even smaller than) the lower limit to the number of cycles for this toy model with $n \sim 0.1-1.0 \mathrm{~cm}^{-3}$.

\subsubsection{Model II: Line-driven wind}

Here we investigate the possibility that the nebula is produced by the wind driven by UV absorption lines. Because the accretion disk in NGC1313 X-2 is relatively cool $(k T \sim 0.1-0.2 \mathrm{keV})$, its radiation is dominated by $\mathrm{UV} /$ soft $\mathrm{X}$-ray emissions, which can be efficiently absorbed in the nebula. There are plenty of absorption lines in UV spectral range, and their opacity is several orders larger than the opacity for electron scattering. Thus, the line-driven wind mechanism allows for efficient momentum transfer between radiation and the nebula, resulting in fast moving plasma (Lucy \& Solomon 1970).

The size of ionized region can be calculated as the Strömgren radius, within which the rate of recombination balances the emission rate $\dot{N}_{\text {ly }}$ of ionizing photons from the central star. For typical $O$ stars with luminosities $L>10^{4} L_{\odot}$, densities in Strömgren sphere $n>5 \times 10^{3}$ $\mathrm{cm}^{-3}$ and Strömgren radii are $<1$ pc (Habing \& Israel 1979). The emission rate of NGC1313 
X-2 estimated with XSEPC, $\dot{N}_{\mathrm{ly}} \sim 10^{50-51} \mathrm{~s}^{-1}$, is $1-2$ orders larger than a typical O star $\left(\sim 10^{49-50} \mathrm{~s}^{-1}\right.$, Martins et al. 2005). Moreover, its surrounding ISM has a much lower number density $n \sim 0.1-1 \mathrm{~cm}^{-3}$, i.e., lower recombination rate. Thus, the UV photons from its accretion disk can reach a large distance and form an ionized zone with a radius of $R_{\mathrm{S}} \approx\left(\frac{3 \dot{N}_{\mathrm{ly}}}{4 \pi \alpha_{\mathrm{H}} n^{2}}\right)^{1 / 3} \approx 140\left(\frac{\dot{N}_{\mathrm{ly}}}{10^{50} \mathrm{~s}^{-1}}\right)^{1 / 3}\left(\frac{n}{1.0 \mathrm{~cm}^{-3}}\right)^{-2 / 3}$ pc (Cen \& Haimen 2000), which agrees with the size of the bubble nebula $\left(R_{\mathrm{bub}}\right)$.

Adopting the spectral profile obtained in Section 2, we obtain that the 0.001-0.3 keV unabsorbed flux is $\sim 1-3$ times of $0.3-10.0 \mathrm{keV}$ unabsorbed flux. Assuming solar abundances, the bubble nebula with number density $n \sim 0.1-1 \mathrm{~cm}^{-3}$ and the radius $R_{\text {bub }} \sim 100 \mathrm{pc}$ (Pakull \& Mirioni 2002; Pakull et al. 2006), would absorb more than $50 \%-90 \%$ of $0.001-0.3$ $\mathrm{keV}$ emission. The line-driven wind scenario also depends on the absorption column density, the metal abundances of the bubble nebula, and the cumulative radiation energy in UV/soft X-ray band, etc. The low metallicity of the bubble nebula (e.g., Hadfield \& Crowther 2007; Zampieri \& Roberts 2009) should decrease the momentum transfer efficiency between the radiation and the interstellar medium. On the other hand, the UV emission would be enhanced because of X-ray reprocessing at outer disk (Tao et al. 2011). Therefore it is highly plausible that at least several percents of the total radiation energy of NGC1313 X-2 is absorbed and stored in the bubble nebula.

If NGC1313 X-2 has been active for $\sim 10^{5} \mathrm{yr}$ with $L \sim 10^{40} \mathrm{erg} / \mathrm{s}$, only a few percents of cumulative radiation energy $\left(\sim 3 \times 10^{52} \mathrm{erg}\right)$ transferred to the diffuse interstellar medium is sufficient to fully ionize and power the expansion of the bubble nebula. We thus conclude that this bubble nebula is very likely produced by photoionization and line-driven expansion. This avoids the conflict between the short accretion age and the apparently much longer bubble age inferred from the expansion velocity of the nebula, since the line-driven expansion happens almost instantaneously everywhere in the nebula.

If NGC1313 X-2 is a transient, there are two possible cases of ionizing the nebula in the line-driven wind scenario. Case I: If the radiation energy in the last active cycle is larger than $E_{\text {bub }}$, the bubble nebula is created in the last active cycle with only $\sim 10 \%$ of the energy required by the radiatively driven outflow scenario. Case II: If the radiation energy in the last active cycle is less than $E_{\text {bub }}$ and the quench time scale $\tau_{\text {quench }}$ is less than the cooling time scale $\tau_{\text {cool }}$, the radiation energy input to the whole bubble nebula can be accumulated during the previous active phases and the whole nebula is speeded up gradually, since the radiation can reach the whole nebula almost instantaneously. As a comparison, the radiatively driven outflow scenario needs the input energy for each cycle to be larger than $E_{\text {bub }}$ because the input energy during the previous cycles cannot be accumulated in the whole nebula, due to the limited shock propergation velocity (Section 4.3.1). Thus, the line-driven wind scenario 
is preferred since the energy required by this model can be much more easily satisfied in all possible scenarios.

\section{Conclusion}

In this study, we present both spectral and timing analyses of NGC1313 X-2 with XMMNewton archive data, together with the information of surrounding ionized bubble, and make the conclusion as follows.

(1) The accretion disk of NGC1313 X-2 is truncated at a large distance $\sim 50 R_{\mathrm{ISCO}}$, and draws the similar evolution pattern to luminous Galactic XRBs. If the inner disk leaves the $R_{\mathrm{ISCO}}$ with increasing luminosity from $\sim 0.3 L_{\mathrm{Edd}}$, the fitting results imply that the central $\mathrm{BH}$ has a fast retrograde spin.

(2) In the super-critical accretion, the radiative and mechanical energy conversion efficiency is very low, that is, even lower than the radiative efficiency of the nuclear fusion $\sim 0.007-0.009$. Therefore, the high luminosity indicates that the companion star feeds the BH with an accretion rate $\dot{M} \sim 1 \times 10^{-4} M_{\odot} /$ yr, which lasts less than $10^{5} \mathrm{yr}$. We now are seeing an ephemeral feast in the source. If MXBs in the early universe have the same short-lived, low energy conversion efficiency accretion as NGC1313 X-2, they produce only limited feedbacks on their environments.

(3) The UFOs accompanied with the super-critical accretion might be over ionized and the absorption line might be hidden in super-Eddington X-ray emission. If UFOs are clumpy or anisotropic, we can still expect weak and broad iron line features with the extremely high energy resolution X-ray mission Astro- $H$ in near future.

(4) If the bubble is shock-excited and the velocity of the blastwave is equal to the velocity inferred from the optical emission line $\left(\sim 100 \mathrm{~km} \mathrm{~s}^{-1}\right)$, the apparent bubble age would be up to $\sim 10^{6}$ yr (Pakull \& Mirioni 2002; Pakull et al. 2006), that is much longer than the lifetime of the X-ray active phase $\left(<10^{5} \mathrm{yr}\right)$. It may indicate that the source is a transient with a duty cycle of activity of $\sim$ a few percent, and the bubble nebula is resulted from the radiatively driven outflow formed in different epochs. However, the upper limit to the number of cycles is close to (or even smaller than) the lower limit to the number of cycles for this toy model with $n \sim 0.1-1.0 \mathrm{~cm}^{-3}$.

(5)The Strömgren radius of NGC 1313 X-2 agrees with the size of its bubble nebula. Our calculations thus show that it is very likely that the bubble nebula is photoionized and its expansion is line-driven, by the strong $\mathrm{UV} /$ soft $\mathrm{X}$-ray emission of the cool disk from 
NGC 1313 X-2, which requires much less energy than that required by the radiatively driven outflow scenario and avoids the conflict between the short accretion age and the apparently much longer bubble age.

We thank the referee for a thoughtful review. S.S.W. thanks Weimin Gu, Wei Cui, and Yuan Liu for many valuable suggestions. This work is partially supported with funding by the 973 Program of China under grant 2009CB824800, by the National Natural Science Foundation of China under grant Nos. 11133002, 10725313, 11003018, 11373036 and 11303022 , and by the Qianren start-up grant 292012312D1117210. S.S.W. is funded by the TÜBİTAK Co-funded Brain Circulation Scheme Co-Circulation program.

\section{REFERENCES}

Abolmasov, P., Fabrika, S., Sholukhova, O., \& Afanasiev, V. 2007, Astrophysical Bulletin, 62,36

Abramowicz, M. A., Czerny, B., Lasota, P., \& Szuszkiewicz, E. 1988, ApJ, 332, 646

Bachetti et al. 2013, ApJ in press (arXiv:1310.0745)

Bardeen, J. M., Press, W. H., \& Teukolsky, S. A. 1972, ApJ, 178, 347

Begelman, M. C. 2002, ApJ, 568, L97

Begelman, M. C., King, A. R., \& Pringle, J. E. 2006, MNRAS, 370, 399

Belczynski, K., Bulik, T., Fryer, C. L., et al. 2010, ApJ, 714, 1217

Cen, R., \& Haiman, Z. 2000, ApJ , 542, L75

Collin, S., \& Kawaguchi, T. 2004, A\&A, 426, 797

Cooke, R., Bland-Hawthorn, J., Sharp, R., \& Kuncic, Z. 2008, ApJ , 687, L29

Crenshaw, D. M., \& Kraemer, S. B. 2012, ApJ, 753, 75

Davis, S. W., Blaes, O. M., Hubeny, I., \& Turner, N. J. 2005, ApJ, 621, 372

Fabbiano, G., \& Trinchieri, G. 1987, ApJ, 315, 46

Fabrika, S. N., 2004, Astrophys. Space Phys. Rev., 12, 1 
Farrell, S. A., Webb, N. A., Barret, D., Godet, O., \& Rodrigues, J. M. 2009, Nature, 460, 73

Fender, R., \& Belloni, T. 2012, Science, 337, 540

Feng, H., \& Kaaret, P. 2006, ApJ, 650, L75

Feng, H., \& Kaaret, P. 2007, ApJ, 660, L113

Feng, H., \& Soria, R. 2011, New Astron. Rev., 55, 166

Fragos, T., Lehmer, B., Tremmel, M., et al. 2013, ApJ, 764, 41

Gierliński, M., Done, C., \& Page, K. 2008, MNRAS, 388, 753

Gilfanov, M. 2010, The Jet Paradigm (Lecture Notes in Physics), Vol. 794, ed. T. Belloni (Berlin: Springer), 17

Gladstone, J. C., Roberts, T. P., \& Done, C. 2009, MNRAS, 397, 1836

Goodall, P. T., Alouani-Bibi, F., \& Blundell, K. M. 2011, MNRAS, 414, 2838

Grisé, F., Pakull, M. W., Soria, R., Motch, C., Smith, I. A., Ryder, S. D., \& Böttcher, M. 2008, A\&A, 486, 151

Gu, W.-M., \& Lu, J.-F. 2007, ApJ, 660, 541

Gu, W.-M. 2012, ApJ, 753, 118

Habing, H. J., \& Israel, F. P. 1979, ARA\&A , 17, 345

Hadfield, L. J., \& Crowther, P. A. 2007, MNRAS, 381, 418

Heger, A., Fryer,C. L.,Woosley, S. E., Langer, N., \& Hartmann, D. H. 2003, ApJ, 591, 288

Heil, L. M., Vaughan, S., \& Roberts, T. P. 2009, MNRAS, 397, 1061

Justham, S. \& Schawinski, K. 2012, MNRAS, 423, 1641

Kaaret, P., Ward, M. J., \& Zezas, A. 2004, MNRAS, 351, L83

Kajava, J. J. E., \& Poutanen, J. 2009, MNRAS, 398, 1450

King, A. L., Miller, J. M., Raymond, J., et al. 2012, ApJ, 746, L20

Kubota, A., Tanaka, Y., Makishima, K., et al. 1998, PASJ, 50, 667

Liu, J. F., Bregman J., \& McClintock, J., 2009, ApJ, 690, L39 
Liu, J. F., Bregman, J. N., Miller, J., \& Kaaret, P. 2007, ApJ, 661, 165

Liu, J. F., Orosz, J., \& Bregman, J., ApJ, 2012, 745, 89

Liu, Q. Z., \& Mirabel, I. F. 2005, A\&A, 429, 1125

Lucy, L.B., \& Solomon, P.M. 1970, ApJ, 159, 879

Marshall, H. L., Canizares, C. R., \& Schulz, N. S. 2002, ApJ, 564, 941

Martins, F., Schaerer, D., \& Hillier, D. J. 2005, A\&A , 436, 1049

McClintock, J. E., Shafee, R., Narayan, R., et al. 2006, ApJ, 652, 518

Méndez, B., Davis, M., Moustakas, J., Newman, J., Madore, B. F., \& Freedman, W. L. 2002, AJ, 124,213

Middleton, M., \& Done, C. 2010, MNRAS, 403, 9

Middleton, M. J., Sutton, A. D., Roberts, T. P., Jackson, F. E., \& Done, C. 2012, MNRAS, 420,2969

Miller, J. M. 2007, ARA\&A, 45, 441

Mirabel, I. F., Dijkstra, M., Laurent, P., Loeb, A., \& Pritchard, J. R. 2011, A\&A, 528, 149

Mizuno T. et al., 2007, PASJ, 59, 257

Moon, D.-S., Harrison, F. A., Cenko, S. B., \& Shariff, J. A. 2011, ApJ, 731, L32

Neilsen, J., Petschek, A. J., \& Lee, J. C. 2012, MNRAS, 421, 502

Neilsen, J., Remillard, R. A., \& Lee, J. C. 2011, ApJ, 737, 69

Ohsuga, K., \& Mineshige, S. 2011, ApJ, 736, 2

Okuda T., Teresi V., Toscano E., \& Molteni D., 2005, MNRAS, 357, 2950

Pakull, M. W., Grisé, F., \& Motch, C. 2006, in IAU Symp. 230, Populations of High Energy Sources in Galaxies, ed. E. J. A. Meurs \& G. Fabbiano (Cambridge: Cambridge Univ. Press), 293

Pakull, M. W., \& Mirioni, L. 2002, astro-ph/0202488

Pakull, M. W., Soria, R. \& Motch, C. 2010, Nature, 466, 209 
Patruno, A., \& Zampieri, L. 2008, MNRAS, 386, 543

Patruno, A., \& Zampieri, L. 2010, MNRAS, 403, L69

Pintore, F., \& Zampieri, L. 2012, MNRAS, 420, 1107

Ponti, G., Fender, R. P., Begelman, M. C., Dunn, R. J. H, Neilsen, J., \& Coriat, M. 2012, MNRAS, 422, 11

Power, C., James, G. F., Combet, C., et al. 2013, ApJ, 764, 76

Poutanen, J., Lipunova, G., Fabrika, S., Butkevich, A. G., \& Abolmasov, P. 2007, MNRAS, 377,1187

Punsly, B. 2011, ApJ, 728, L17

Ramsey, C. J., Williams, R. M., Gruendl, R. A., et al. 2006, ApJ, 641, 241

Reynolds, C. S., \& Nowak, M. A. 2003, Phys. Rep., 377, 389

Shakura, N. I., \& Sunyaev, R. A. 1973, A\&A, 24, 337

Soria, R. 2007, Ap\&SS, 311, 213

Soria, R. 2011, Astronomische Nachrichten, 332, 330

Steiner, J. F., Narayan, R., McClintock, J. E., \& Ebisawa, K. 2009, PASP, 121, 1279

Stobbart, A. M., Roberts, T. P., \& Wilms, J. 2006, MNRAS, 368, 397

Strohmayer, T. E., \& Mushotzky, R. F. 2003, ApJ, 586, L61

Swartz, D. A., Soria, R., Tennant, A. F., \& Yukita, M. 2011, ApJ, 741, 49

Tao, L., Feng, H., Grisé, F., \& Kaaret, P. 2011, ApJ, 737, 81

Tombesi, F.,Cappi, M., Reeves, J. N., \& Braito, V. 2012, MNRAS, 422, L1

Walton D. J., Miller J. M., Reis R. C., \& Fabian A. C., 2012, MNRAS, 426, 473

Webb, N., et al. 2012, Science, 337, 554

Weng, S.-S., Wang, J.-X., Gu, W.-M., \& Lu, J.-F. 2009, PASJ, 61, 1287

Weng, S.-S., \& Zhang, S.-N. 2011, ApJ, 739, 42 (Paper I)

Wheeler, J. C., \& Johnson, V. 2011, ApJ, 738, 163 
Yao, Y., Zhang, S. N., Zhang, X., Feng, Y., \& Robinson, C. R. 2005, ApJ, 619, 446

Zampieri, L., Impiombato, D., Falomo, R., Grisé, F., \& Soria, R. 2012, MNRAS, 419, 1331

Zampieri, L., Mucciarelli, P., Falomo, R., et al. 2004, ApJ, 603, 523

Zampieri, L., \& Roberts, T. 2009, MNRAS, 400, 677

Zhang, S. N., Cui, W., \& Chen, W. 1997, ApJ, 482, L155 